\title{
Fiber-Wireless Testbed using Software Defined Radio for Protocol and Algorithm Testing
}

\author{
Mohammad Azmi Ridwan ${ }^{\# 1}$, Nurul Asyikin Mohamed Radzi"², Fairuz Abdullah"\#*3 \\ \#Institute of Power Engineering, Universiti Tenaga Nasional, 43000 Kajang, Selangor \\ * Department of Electrical \& Electronics Engineering, College of Engineering, \\ Universiti Tenaga Nasional, 43000 Kajang, Selangor \\ 11m.azmiridwan@gmail.com, 22asyikin@uniten.edu.my, ${ }^{3}$ fairuz@uniten.edu.my
}

Article History: Received: 10 November 2020; Revised: 12 January 2021; Accepted: 27January 2021; Published online: 05April 2021

\begin{abstract}
Fiber-Wireless (FiWi) network is able to provide abundant bandwidth capacity and mobility to the end-users. It also eliminates the needs of having complete tedious end-to-end fiber installation from the central office to the users which saves tremendous capital expenditure. However, FiWi is still progressing. Researchers worldwide are still developing experimental works for improvement on the network reliability, quality-of-services and security. Almost all recently proposed testbed designed for FiWi are using hardware that lacks in programmability feature, making it difficult to implement any protocols and algorithms. A testbed must be flexible, scalable and reprogrammable so that various experiments and testing can be implemented easily for testing purposes. In this paper, a reprogrammable FiWi testbed using software defined radio (SDR) is proposed. One of the most prominent SDR available in the market is Universal Software Radio Peripheral (USRP). It is chosen to be used in this paper as it is equipped with a user-friendly programming platform; LabVIEW. To test the testbed's reprogrammability feature, two algorithms are implemented for proof-of-concept; collision avoidance and dynamic bandwidth allocation. The collision avoidance algorithm is implemented in the wireless side of the testbed using the concept of Carrier Sensing Multiple Access/Collision Avoidance. At the fiber domain, a dynamic bandwidth allocation-limited scheduling is incorporated in the testbed. The results show that algorithms implemented in the testbed are in-line with the expected results. It proves that the testbed can be used for future algorithm testing for research purposes.
\end{abstract}

Keywords:Fiber-Wireless, Software Defined Radio, Passive Optical Network, Reprogrammable, LabVIEW

\section{Introduction}

The telecommunication networks are becoming more congested with the ever-increasing number of Internet subscribers consuming the available resources. It is forecasted that the Internet traffic can reach 3.3 zettabyte annually by 2021 that is corresponding to 3 -fold increase from 2016 [1].

It is more exacerbated by the bold needs of ensuing generation wireless technology like 5G demanding improvement across all metrices. With that, network providers are improving the current network architecture to cater the demand.

It is well-known that fiber optics offers huge bandwidth capacity, energy savings, service transparency, low propagation loss and improved security. Passive transmission of optical signal in passive optical network's (PON's) architecture ensures its high reliability without electromagnetic interference [2]. However, end-to-end fiber installation from the service provider to each subscriber requires tedious installation works and not cost effective.

Wireless network on the other hand offers more flexibility at a lower cost. It allows users to stay connected without being tethered into a wired connection. But it has limited bandwidth and vulnerable to interference. To overcome the shortcomings from fiber and wireless domain, the integration of Fiber-Wireless (FiWi) network was introduced. Fiber network is one of the solutions for the limited bandwidth and vulnerability issues from the wireless network and in addition to that, wireless network can provide mobility to the entire users.

FiWi is still an on-going research for further improvement especially with the upcoming $5 \mathrm{G}$ communications demanding improved FiWi architecture. A lot of revolutionary works are required on the existing network platform in combination with new photonics technologies and wireless radio access platform to address the current limitations [3]. Besides, there are also room for improvement in terms of security and survivability, which has becoming the primary consideration of the major operators [4]. With that said, a FiWi testbed is very crucial to perform testing to ensure that it will be fully functional when it is implemented in the real world. The testbed should be flexible, scalable and reprogrammable to any protocols so that the Service Level Agreement can be experimentally tested and validated. 
The use of programmable radio is promising for FiWi testbed as it can transfer information from transmitter to a receiver. Software-controlled radio comes with a built-in pre-programmed radio, while software defined radio (SDR) is a reprogrammable radio that performs signal processing, signal modulations and demodulations in a single unit. The implementation of protocols or algorithms in the SDR can be easily done via programming.

To date, there are numerous researchers world-wide developing FiWi testbed to test new technologies and schemes to further improve the current FiWi networks. For instance, Li et al. [2] demonstrated a bidirectional FiWi system via a testbed to transmit signals with different fiber capacities and wireless frequencies. The testbed has successfully demonstrated multiple fiber capacities and wireless frequencies with an efficient bit-error-rate (BER) over the 40-km SMF with 10, 25 and $100 \mathrm{~m}$ of wireless distance. Comparable to SDR, the wireless frequencies are tuneable within a range that is up to the researcher's preference. However, SDR offers another important feature that is reprogrammability where any protocols and algorithms can be programmed into the SDR for Quality of Service (QoS) testing purpose.

Omar et al. [5], experimentally demonstrated the generation and transmission of millimetre wave signal using photonics technology via a FiWi testbed. They reported a microwave photonics-based system for 5G signal distribution and transmission over hybrid optical and wireless links. The proposed FiWi testbed successfully demonstrated that the setup of $10 \mathrm{~km} \mathrm{SMF}, 6.5 \mathrm{~m}$ free-space optical and $1 \mathrm{~m}$ radio frequency channel has a BER value below the error vector magnitude limit. However, the FiWi testbed in [5] lacks the reprogrammability features.

To overcome noises and distortions in the FiWi network, an intensity modulation-direct detection scheme using external modulator is proposed by Gonzalez et al [6]. They proposed a radio-over-fiber Xbee transmissionbased testbed using MZ modulator, polarization controller, microwave signal generator and among others. The data issued from two XBee modules are coded on a wireless microwave carrier at $2.44 \mathrm{GHz}$ were transmitted through an optical link of $25 \mathrm{~km}$. Proposed testbed uses external modulator and XBee as the equipment to transmit data. Compared to SDR, the modulation and wireless transceiver is embedded in a single unit making experimental works more convenient.

Christina et al. [7] developed a FiWi testbed to experimentally compare the different transport schemes incorporating modified double-sideband suppressed carrier (DSB-SC) and optical single sideband with carrier (OSSB) modulation formats to cater the transport of $60 \mathrm{GHz}$ wireless signals. The proposed testbed uses MATLAB to generate WAN signals. Madry et al. [8] proposed a FiWi-based sensor system for temperature monitoring using Sagnac-Loop Interferometer integrated with Arduino and ZigBee. The proposed sensor network uses a microcontroller; Arduino Nano which has the reprogrammability features. However, it may not have the capability to perform tuneable wireless frequency, modulation, or generation of RF signals on its own.

Dat et at. [9] developed a proof-of-concept experimental setup to demonstrate high-speed and uninterruptable communications between central stations on a ground to a base station on high speed train using switched wavelength-division multiplexing FiWi backhaul networks. The testbed successfully transmitted approximately 20 and 10 Gbps signals over the switched FiWi links in the downlink and uplink directions respectively. The proposed testbed is claimed to provide high-speed and advanced services for fast-moving users for future 5G and beyond networks.

All the mentioned related works are mostly pure hardware based FiWi testbed which requires tedious hardware setups that may be hard to configure, making it less suitable for architecture and protocol testing. As FiWi is still developing, the best architecture, algorithms and protocols are scrutinised to improve the network overall performance. Therefore, it is essential to develop a reprogrammable testbed to observe the performance of various FiWi networks in terms of QoS.

In this paper, a FiWi testbed is proposed using SDR that is able to be reprogrammed to test the FiWi network's QoS. This paper is organised as follows; material and method is discussed in Section 2, the results and discussions are discussed in Section 3. Finally, the conclusion and future works is summarised in Section 4.

\section{Material and Method}

In this section, the design of the SDR-based FiWi testbed is discussed. One of the most prominent SDRs is developed by National Instrument's Universal Software Radio Peripheral (USRP). The multi-purpose processor of the USRP allows the reuse of the hardware for different product through programming [10]. Aside from the 
reprogrammable features offered by USRP, it is also useful for efficient and real time realization of wireless systems operation in RF band [11] and can use real signal data to perform various testing [12].

There are several USRPs available in the market such as USRP RIO and FlexRIO which are equipped with global positioning system. All the available USRPs are programmable but they are different in terms of the frequency range, instantaneous bandwidth, ability to transmit and receive simultaneously as well as its oscillator type.

For the proposed FiWi testbed, the wireless frequency selected is $2.4 \mathrm{GHz}$ which is equivalent to WiFi's frequency for proof-of-concept and it requires a simultaneous transmit and receive feature. USRP-2922 is chosen since it suits the requirement mentioned before. Four sets of USRP-2922, namely SDR 1-SDR 4 are used in the proposed testbed. The chosen FiWi architecture is the PON which consist of optical line terminal (OLT), optical network units (ONU) and end-user. Connection between SDR to ONUs is fiber link while ONUs to end-users are done wirelessly.

The hardware structure is illustrated in Fig. 1 with architecture ratio of $(1: 2: 1)$ where it consists of 1 OLT, 2 ONUs and 1 end-user. SDR 1 represents OLT, SDR 2 and SDR 3 represent ONU 1 and ONU 2 respectively

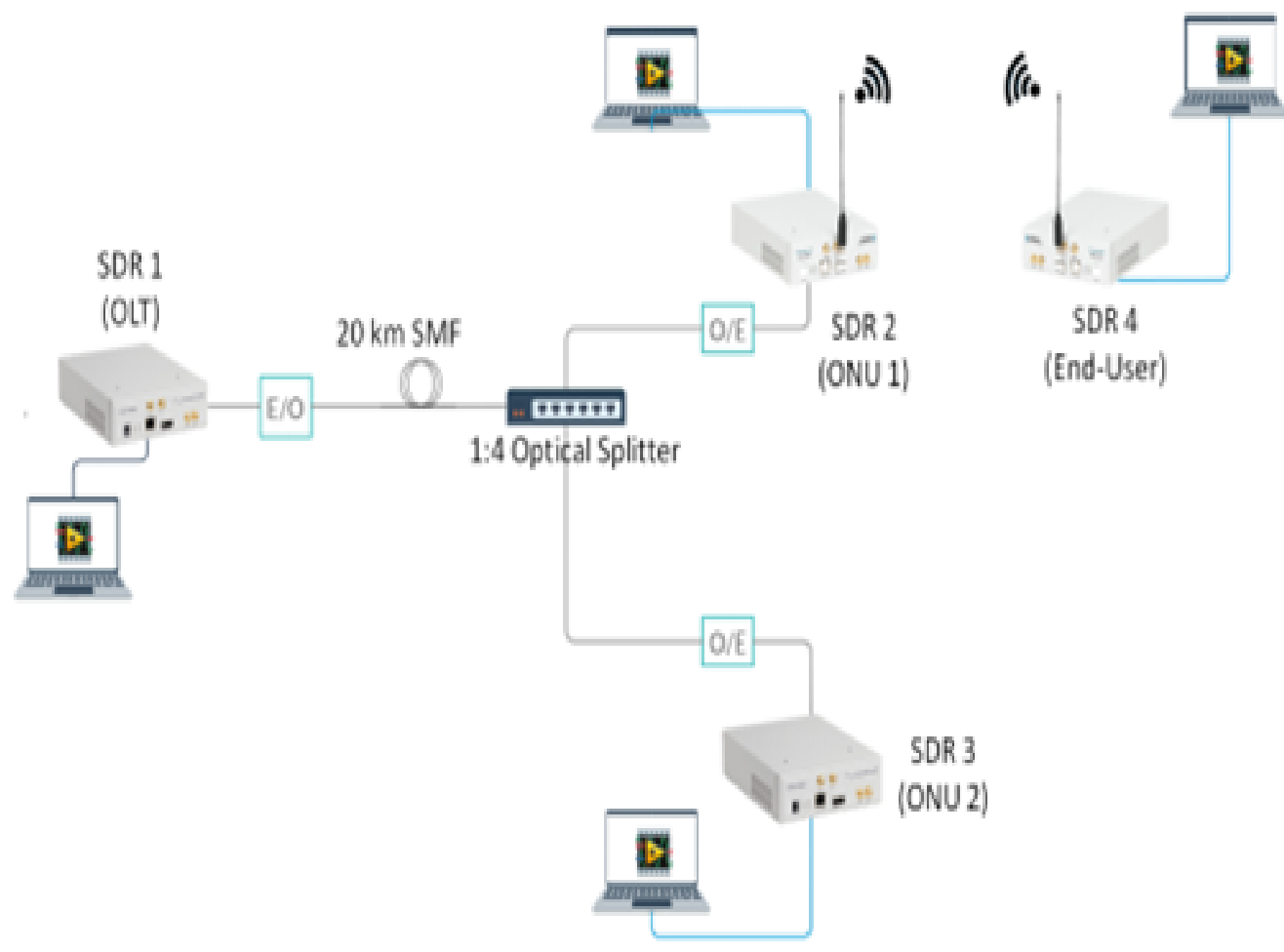

Figure 1. Proposed FiWiTestBed

while SDR 4 represents the end-user. With the flexibility of the SDR, the architecture can be change to other ratios such as $(1: 1: 2),(2: 1: 1)$ or other ratios according to the researcher's preference with minimal hardware and software re-arrangement.

SDR 1 is connected to the electrical to optical (E/O) converter via SubMiniature Version A (SMA) cable. E/O converter is essential in this testbed as it converts the output of the SDR into optical signals via radio-offiber (RoF) technology. Then, the optical signals travel via $20 \mathrm{~km}$ single mode fiber (SMF) to the 1:4 optical splitter where the signals are split to SDR 2 and SDR 3. The optical signals are reverted to RF signals using the optical to electrical (O/E) converter. Finally, the RF signals is transmitted to SDR 4 wirelessly via a vertical gain antenna. When a SDR receives a signal, it will be down converted, sampled and streamed to the computer via 1 $\mathrm{Gb}$ Ethernet cable. Fig. 2 shows the SDR arrangement for the recommended FiWi testbed. 


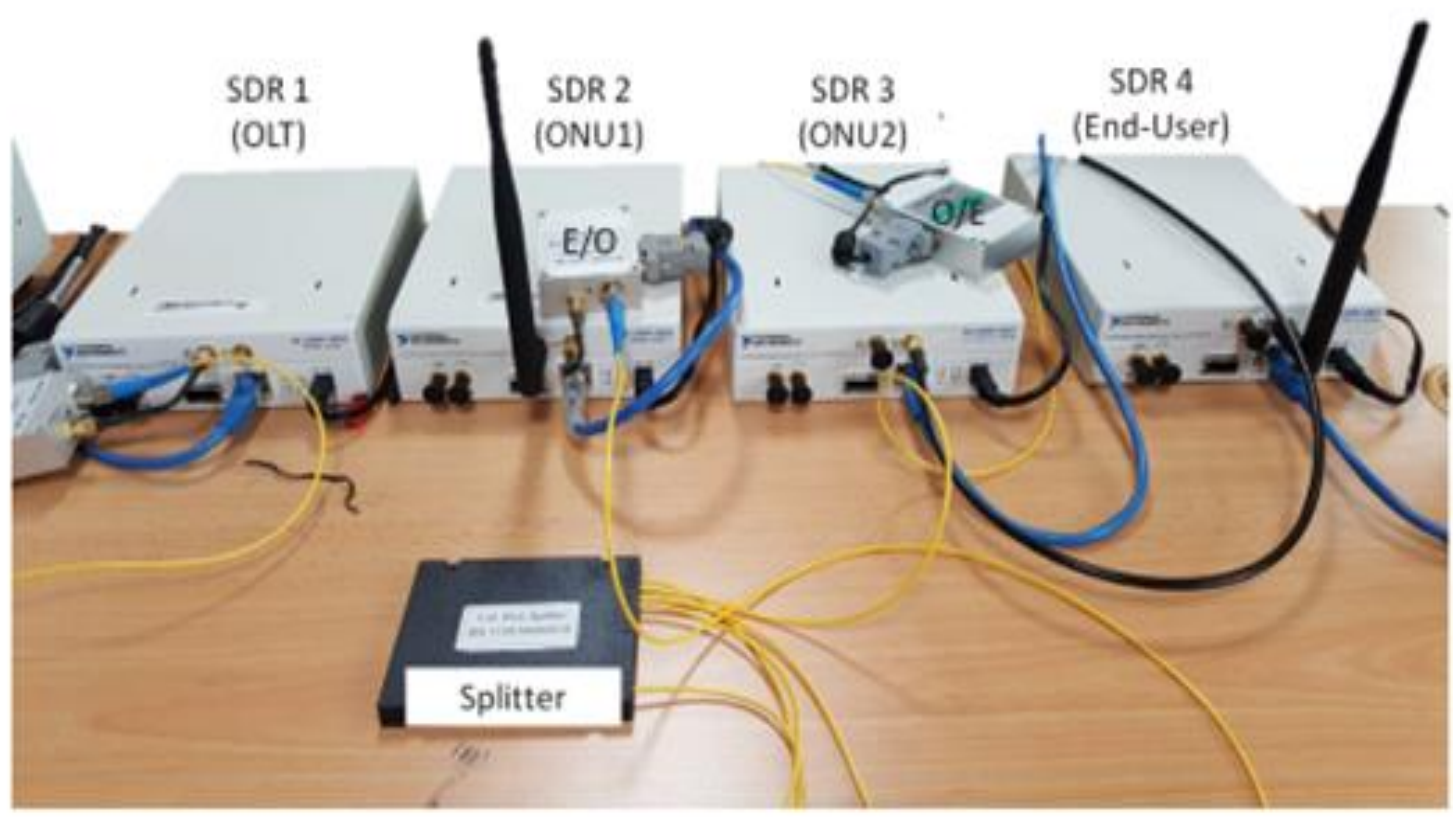

Figure 2.SDR setup for the proposed FiWi testbed

For this testbed, the USRP is integrated with LabVIEW where all the configurations and data source are held. This testbed uses text as data source and user can choose various number of packets to transmit from the userinterface. The user interfaces are illustrated in Fig. 3. User are only required to type the IP address for each SDR and choose the number of packets for transmission from the selection box and click on the start button in LabVIEW. The transmission will start immediately.

The receive and transmit parameters including sampling rate, modulation type, transmission's power gain and wireless frequency are adjustable as per the researcher's preferences, making this testbed user-friendly, without having to perform the tedious hardware reconfigurations. For downstream transmission, the time allocation is transmitted to the ONU from the OLT according to the chosen number of packets to transmit. The ONU will receive the time allocation and is ready to receive the data from OLT only in that allocated time frame. The steps are repeated for transmission at the wireless domain. The process is in reverse for upstream transmission.

LabVIEW offers a great programming platform for researchers as it allows integration of multiple programming languages including math script from MATLAB, C, or graphical programming (G-programing) that is introduced by NI. G-programming is graphical-based programming where it is translated from the line-byline coding. With that, implementations of any algorithms and protocols testing are made simple.

As a proof-of-concept, to test the testbed's reprogrammability feature, two simple algorithms are implemented; carrier sensing multiple access/ collision avoidance (CSMA/CA) and dynamic bandwidth allocation (DBA).

\section{A. CSMA/CA}

One of the collision avoidance protocol in the network is CSMA. With CSMA, it senses for any activity in the network before allocating an approval to transmit. CSMA can be divided into CSMA/CA and CSMA/Collision Detection. Wireless network with IEEE 802.11 standard such as WiFi uses CSMA/CA as collision avoidance protocols. Each nodes in the network is granted with equal probability in accessing the network.

The CSMA/CA is incorporated for upstream transmission as illustrated in Fig. 4. By default, multiple endusers are present for architecture (1:1:2), both end-users are granted equal opportunity to transmit in round-robin fashion. However, there will be a possibility when multiple end-users transmit data simultaneously. Hence, causing a collision. When this occurs, the CSMA/CA algorithm will prevent both end-users to transmit data 
simultaneously, causing collision and data corruptions. The moment the algorithm detects multiple simultaneous transmissions, the program will halt all transmissions. With the assumption that both end-users have the same priority, the end-users will transmit in sequence. As a result, no collision occurs. After both end-users transmitted all their intended packets, it will run as usual unless simultaneous transmission is detected again. The flowchart for the CSMA/CA implemented is as shown in Fig. 5.

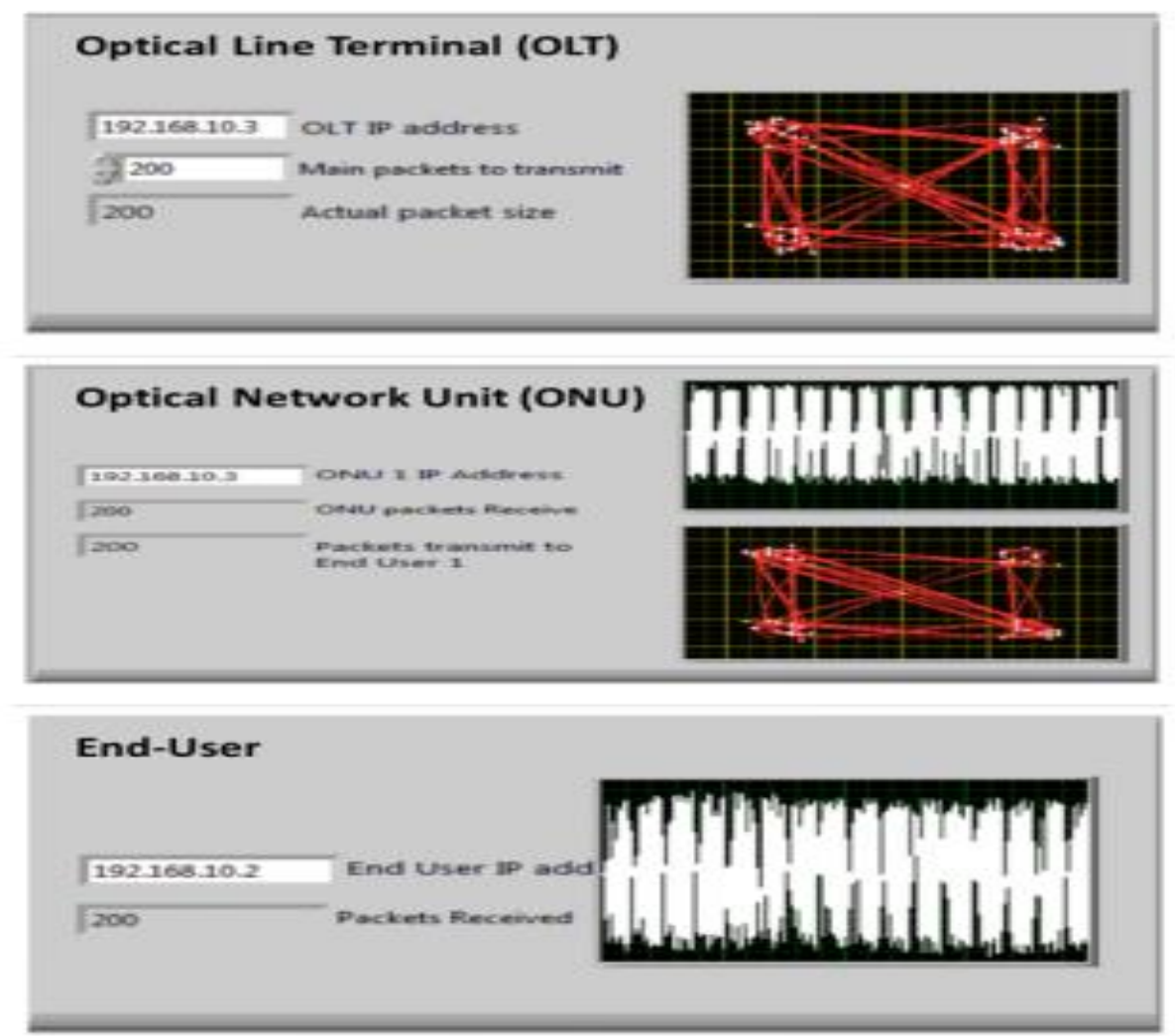

Figure 3. User-Interface for OLT, ONU, and End-User

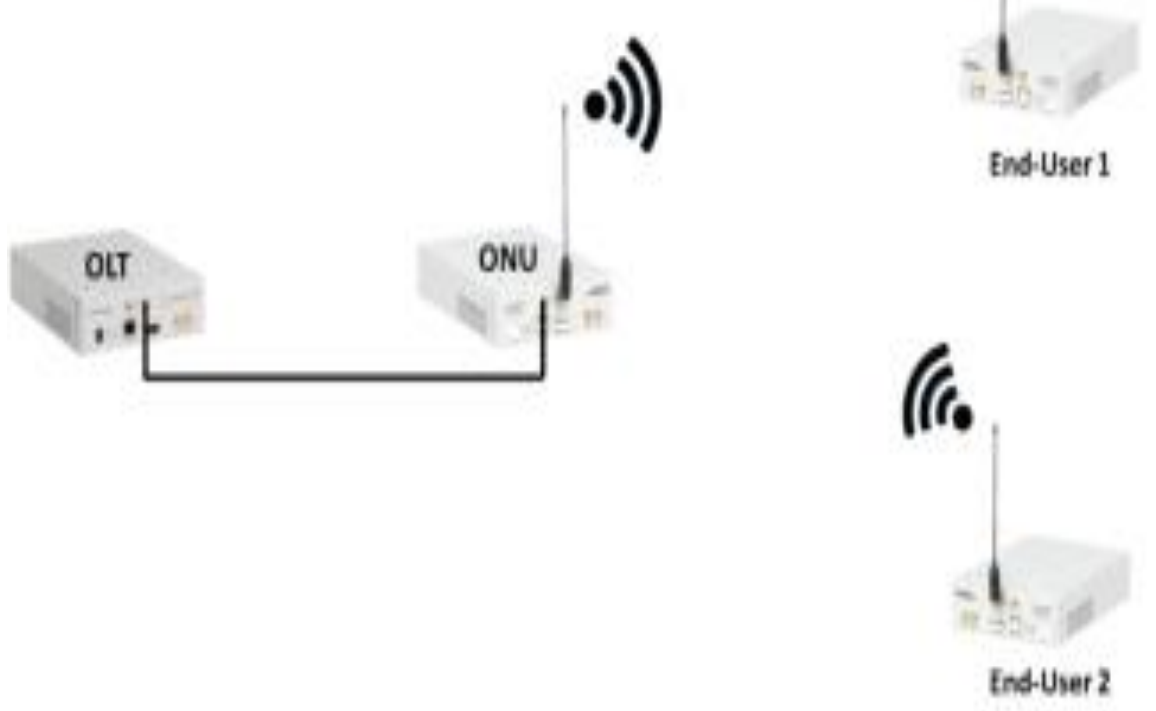

Figure 4. Hardware configuration for CSMA/CA protocol 


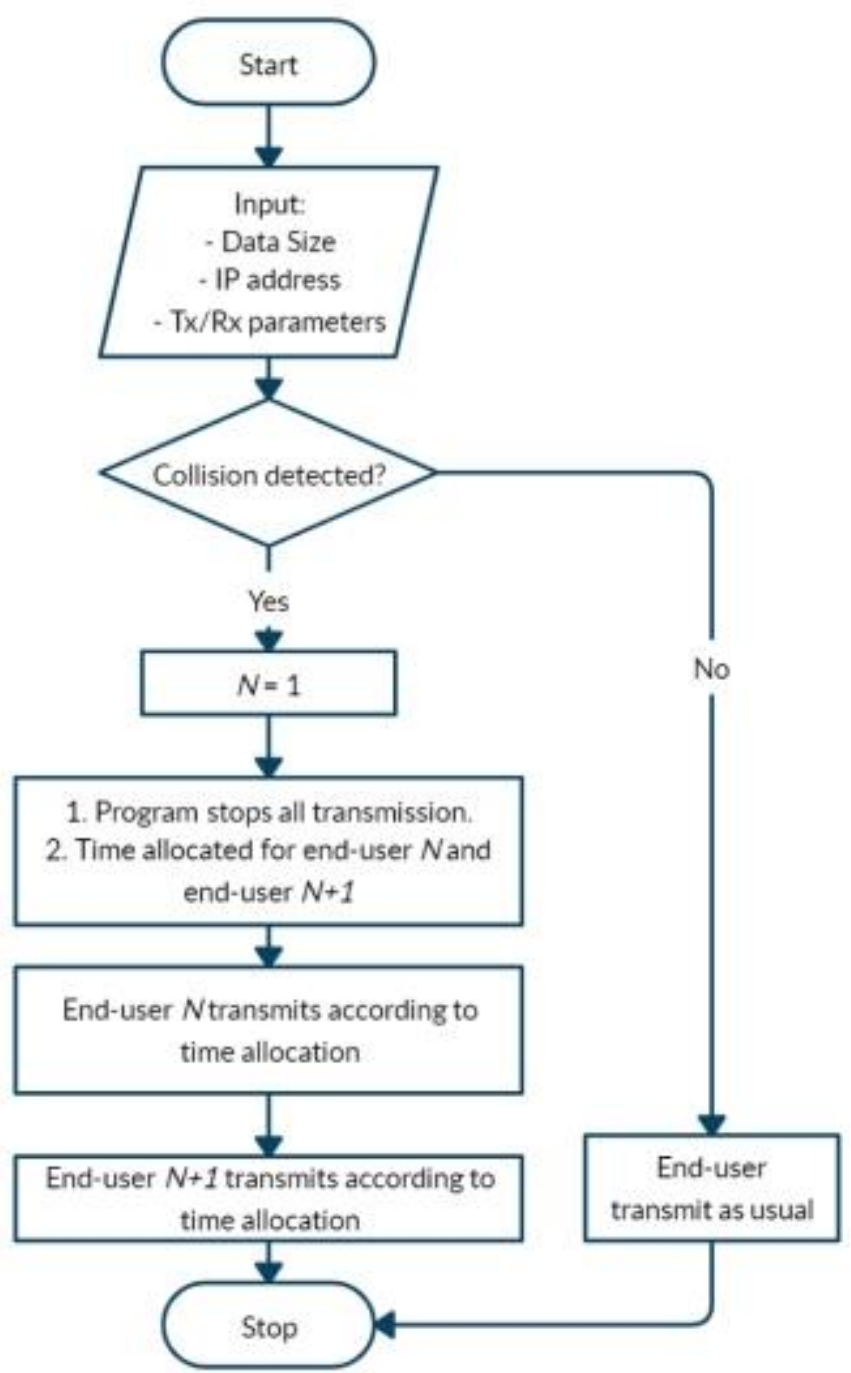

\section{B. $\boldsymbol{D B A}$}

Figure 5. Flowchart for the CSMA/CA algorithm

The second algorithm implemented in the SDR-based testbed is the DBA-Limited Scheduling.The bandwidth utilization and propagation delay can be improved by dynamically allocating the bandwidth as per request [13]. The DBA algorithm is implemented in the fiber domain. Each ONU in the network will be granted with bandwidth based on:

$$
B_{g \text { (packets) }}=\left\{\begin{array}{c}
B_{\text {req }}, B_{\text {req }} \leq W_{\text {max }} \\
W_{\text {max }}, B_{\text {req }}>W_{\text {max }}
\end{array}\right.
$$

where $B_{g(\text { packets })}$ is the bandwidth allocated for the ONUs in the form of number of packets. $B_{\text {req }}$ is the demanded bandwidth of the ONUs to the OLT. $\mathrm{W}_{\max }$ is the maximum bandwidth allocated for each of the ONUs in the network which is determined as follows:

$$
W_{\max }=R_{u} \times\left(\frac{T_{\max }}{N}-B\right)
$$

If the $B_{r e q}$ is less than the $W_{\max }$, the granted bandwidth is as per requested. However, if $B_{r e q}$ is greater than $W_{\max }$, the bandwidth granted for the ONU is capped at $W_{\max } . T_{\max }$ is the maximum cycle time, B is the guard time, $R_{u}$ is the upstream bandwidth and $N$ is the number of ONU in the network. For this algorithm, the standard of Ethernet PON is chosen where the value of $T_{\max }, B, R_{u}$, and $N$ is $2 \mathrm{~ms}, 5 \mu \mathrm{s}, 1 \mathrm{Gbps}$ and 2 respectively. However, as mentioned previously, the bandwidth for the USRP 2922 is $40 \mathrm{Mhz}$ for 8-bit sampling, hence, scaling is needed. Scaling technique has also been used by [14-15]. Scaling ratio of 0.08 is used to upscale the 
bandwidth of the USRP to 1Gbps. The flowchart for DBA is depicted in Fig. 6 while the GUI in LabVIEW is as depicted in Fig. 7. The granted bandwidth in Fig. 7 is capped at $100 \%$ which is the $W_{\max }$ when the $B_{r s q}$ is greater than $W_{\max }$.

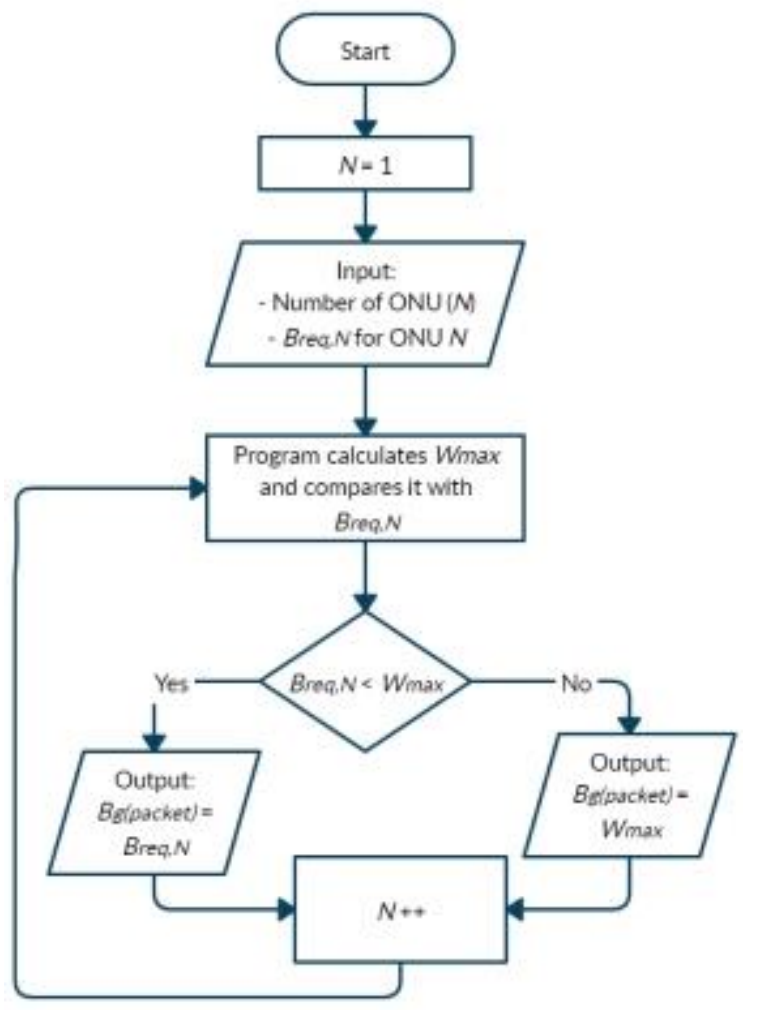

Figure 6. Flowchart for DBA implemented in the proposed FiWi testbed

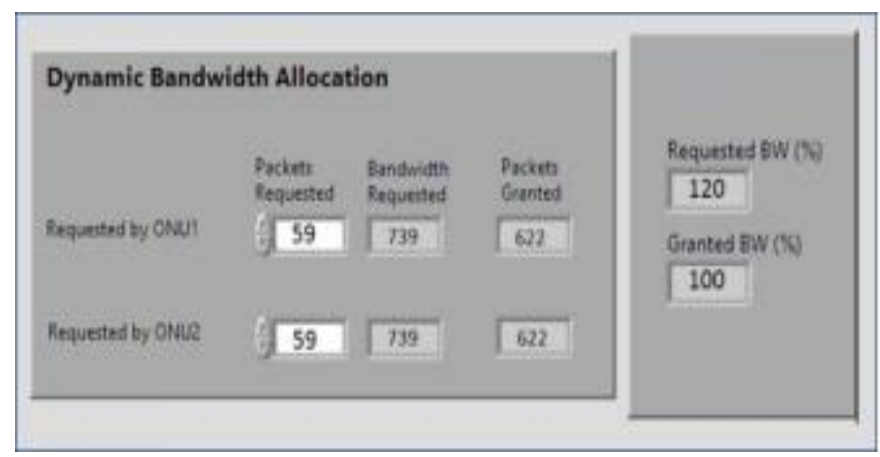

Figure 7. GUI for the DBA algorithm in LabVIEW

\section{Results and Discussion}

In order to verify that the proposed SDR-based FiWi testbed is reprogrammable, the collision avoidance protocol and dynamic bandwidth algorithm are implemented in the testbed. Experiments are performed and the results are discussed in this section.

For the collision avoidance algorithm, the experiment is conducted using case by case method and it is summarized in Table 1. Fig. 8(a) shows the result for Case 1. ONU received all 505 packets within the allocated time frame which is $15 \mathrm{~s}$. After a predetermine rest period, the end-user 2 transmit 2015 packets within the allocated time frame of $20 \mathrm{~s}$. At the end, the ONU received all 2015 packets without any packets loss as expected. A rest period is allocated for the ONU to stabilize after receiving from the first end-user. Experimentally, the ideal "rest" period for the ONU is $3 \mathrm{~s}$. 
Fig. 8(b) shows the result for Case 2 where the end-user 1 transmitted 1009 packets for the first $15 \mathrm{~s}$ and enduser 2 transmit 4028 packets after the $3 \mathrm{~s}$ for $30 \mathrm{~s}$ allocated timeframe. The ONU received all 5026 packets successfully without any no corrupted data. Finally, Fig. 8(c) shows the result for Case 3 with the ONU received all 8056 packets from both end-user 1 and end-user 2 with no reported any loss packets.

The next algorithm is DBA-limited scheduling. The requested bandwidth with a step size of $10 \%$ will be translated into percentage from 0 to $100 \%$. The experiment with DBA is run and the results are as shown in Fig. 9. The calculated and experimental granted bandwidth is perfectly in-lined, proving that the DBA algorithm implemented is fully functional.

Table 1.Case 1, 2 and 3 for CSMA/CA experiment

\begin{tabular}{|c|cc|cc|c|}
\cline { 2 - 5 } \multicolumn{1}{c|}{} & \multicolumn{2}{c|}{ End-User 1 } & \multicolumn{2}{c|}{ End-User 2 } & \multirow{2}{*}{ Expected packets } \\
\hline Case & Packets & Allocated time (s) & Packets & Allocated time (s) & \\
\hline 1 & 505 & 15 & 2015 & 20 & 2520 \\
2 & 1009 & 15 & 4028 & 30 & 5026 \\
3 & 2015 & 20 & 6041 & 40 & 8056 \\
\hline
\end{tabular}

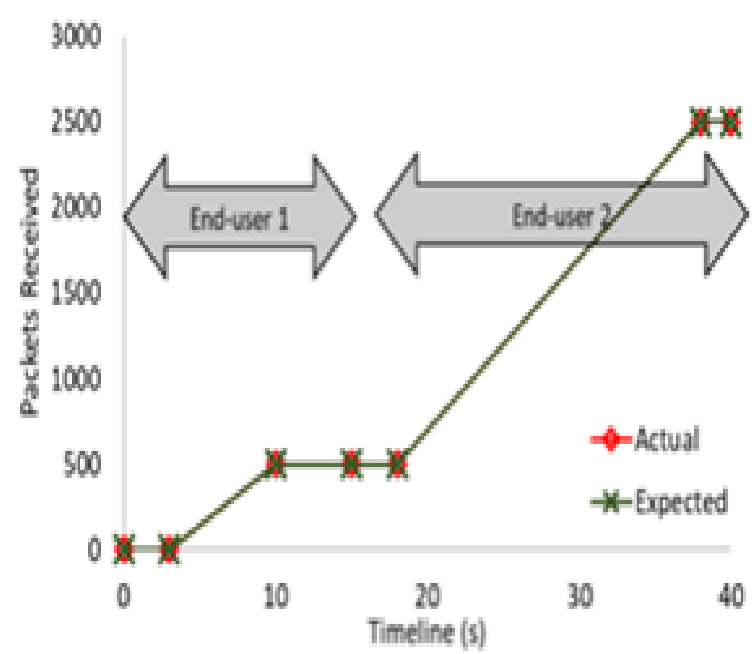

(a)

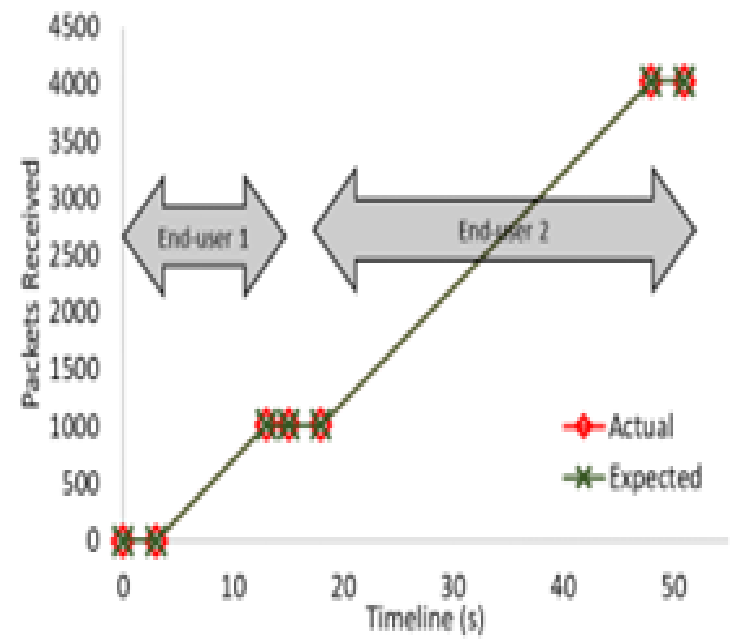

(b)

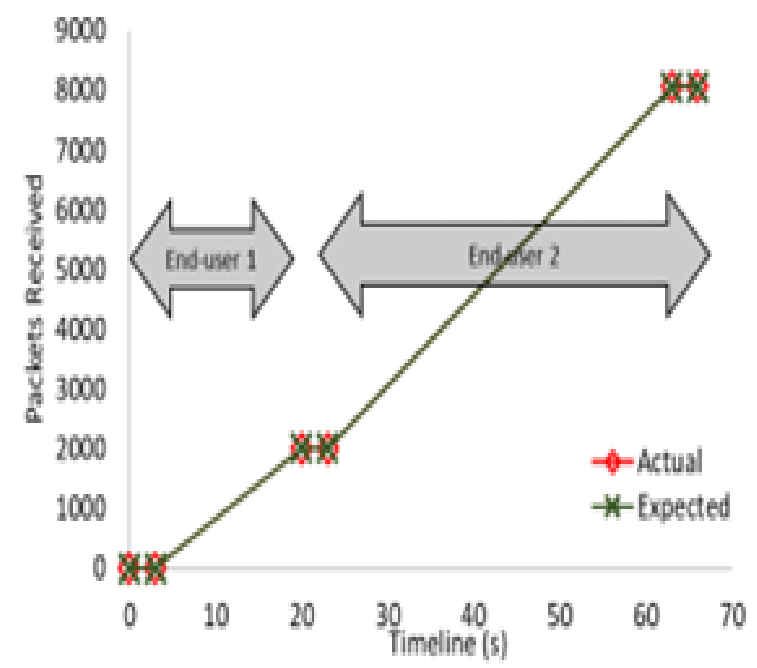

(c)

Figure 8. Total Packets received by the ONU vs Timeline for (a) Case 1 (b) Case 2 (c) Case 3 


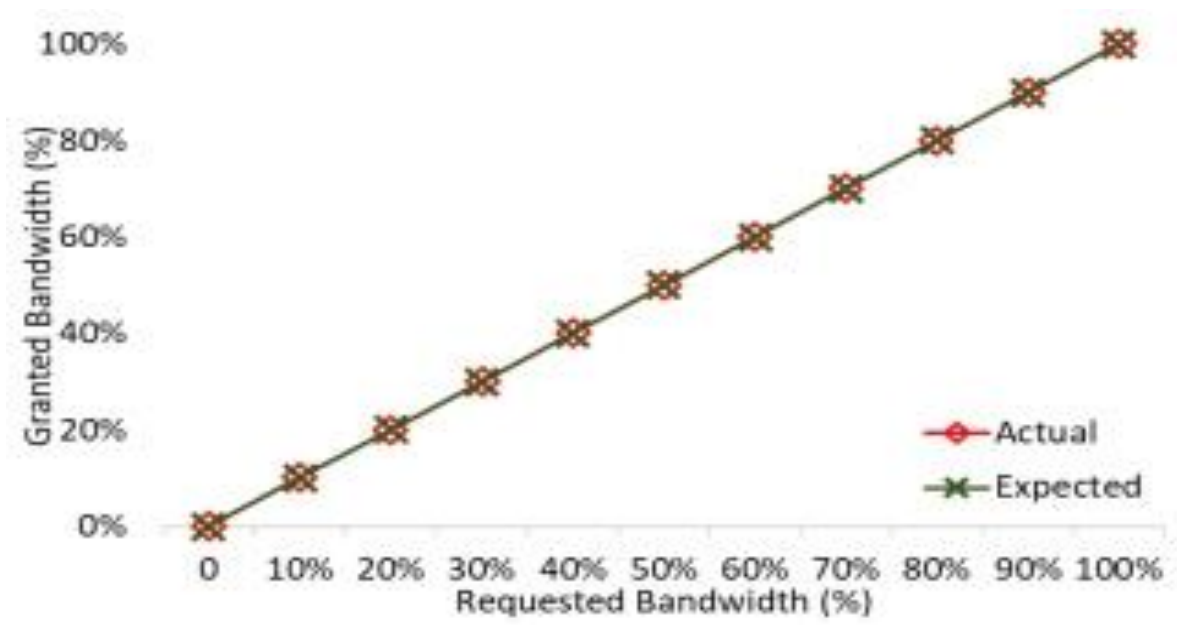

Figure 9. Granted bandwidth vs requested bandwidth for DBA-Limited Scheduling

\section{Conclusion}

Due to the benefits offered by fiber and wireless links, FiWi can be seen as one of the favourable technologies for the next-generation network architecture. Fiber link can feed every growing amount of applications and perpetually increasing bandwidth demand. Concurrently, users can enjoy the mobility and flexibility offered by wireless link. With the communication systems that are currently growing towards the 5G generations, the network demand is expected to keep on increasing. A better system with broader coverage, better utilization of available spectrums, improved data rates and security requires more focus and demand. This paper proposed a FiWi testbed that can be reprogrammable to any algorithms and protocols using the easy-to-use platform that comes with interactive user interface. The proposed testbed is implemented with simple algorithm as a proof-of-concept that the testbed can be reprogrammed easily. The results show that the CSMA/CA and DBA algorithm implemented in the testbed is in-line with the expected results, proving that the proposed testbed can be used for algorithm testing.

\section{Acknowledgment}

We would like to acknowledge and thank the Ministry of Education, for providing fund under Fundamental Research Grant Scheme (FRGS) FRGS/1/2014/ICT03/UNITEN/02/1.

\section{References}

1. K. Chekima, R. Alfred, and K. O. Chin, "Hybridizing Entropy Based Mechanism with Adaptive Threshold Algorithm to Detect RA Flooding Attack in IPv6 Networks," vol. 488, no. August 2018, pp. 172-185, 2018.

2. Y. Li et al., "Fiber-Wireless and Fiber-IVLLC Convergences Based on MZM-OEO-Based BLS," IEEE Photonics J., vol. 8, no. 2, pp. 1-10, 2016.

3. Gasulla, S. García, D. Barrera, J. Hervás, and S. Sales, "Space-Division Multiplexing for FiberWireless Communications," in 19th International Conference on Transparent Optical Networks, 2017, pp. 2-5.

4. H. Tang and Z. X. Zhou, "The design of communication network optical fiber cable condition monitoring system based on distributed optical fiber sensor," in 2018 International Conference on Electronics Technology, ICET 2018, 2018, pp. 97-101.

5. O. Alkhalifah, O. Alrabiah, A. Ragheb, M. A. Esmail, and S. Alshebeili, "Investigation and demonstration of $5 \mathrm{G}$ signal transmission over fiber/FSO/wireless links," in 2017 International Conference on Electrical and Computing Technologies and Applications, ICECTA 2017, 2018, vol. 2018-Janua, pp. 1-4.

6. L. A. Gonzalez-Mondragon, L. J. Quintero-Rodriguez, A. G. Correa-Mena, J. Rodriguez-Asomoza, A. G. Juarez, and I. E. Zaldivar-Huerta, "Performance Evaluation of Transmission between Two Wireless Devices Based on Radio-over-Fiber Technology," in 2018 15th International Conference on Electrical Engineering, Computing Science and Automatic Control, CCE 2018, 2018, pp. 7-10.

7. Lim, Y. Tian, K. L. Lee, and A. Nirmalathas, "Transport schemes for fiber-based fronthaul for transporting $60 \mathrm{GHz}$ wireless signals," Int. Conf. Transparent Opt. Networks, pp. 3-6, 2017.

8. M. Madry, P. Bak, D. Kowalczyk, and E. Beres-Pawlik, "The Fiber-Wireless Sensor System for 
Temperature Monitoring Using Sagnac-Loop Interferometer," in International Conference on Transparent Optical Networks, 2018, no. 1, pp. 1-4.

9. P. T. Dat, A. Kanno, K. Inagaki, F. Rottenberg, N. Yamamoto, and T. Kawanishi, "High-Speed and Uninterrupted Communication for High-Speed Trains by Ultrafast WDM Fiber-Wireless Backhaul System,” J. Light. Technol., vol. 37, no. 1, pp. 205-217, 2019.

10. L. Y. Tang, Z. W. Xia, G. C. Wan, and M. S. Tong, "A Dynamic Detection Method for RFID Strain Sensor Tag Antenna Based on Usrp X300," in Progress in Electromagnetics Research Symposium, 2018, pp. 2061-2065.

11. I. M. Althaf and S. C. Prema, "Covariance and eigenvalue based spectrum sensing using USRP in real environment," in 2018 10th International Conference on Communication Systems and Networks, COMSNETS 2018, 2018, no. 4, pp. 414-417.

12. G. H. Lee, Y. D. Lee, and I. S. Koo, "Convolution Neural Network-Based Spectrum Sensing for Cognitive Radio Systems Using USRP with GNU Radio,” in International Conference on Ubiquitous and Future Networks, ICUFN, 2018, pp. 862-864.

13. G. Kramer, B. Mukherjee and G. Pesavento, "IPACT a dynamic protocol for an Ethernet

14. PON (EPON)", IEEE Commun. Mag., vol. 40, no. 2, pp. 74-80, 2002

15. Hussain, A., Manikanthan, S.V., Padmapriya, T., Nagalingam, M.(2020). Genetic algorithm based adaptive offloading for improving IoT device communication efficiency. Wireless Networks, 26 (4), pp. 2329-2338.

16. C. Assi, Yinghua Ye, S. Dixit and M. Ali, "Dynamic bandwidth allocation for quality-ofservice over ethernet PONs", IEEE J. Select. Areas Commun., vol. 21, no. 9, pp. 1467-1477, 2003

17. T. Padmapriya\& S.V. Manikanthan, "Retracted: Security and Routing protocol for $5 \mathrm{G}$ wireless mobile networks". IJIMT, 2020 